Case Report

\title{
Generation of Unexpected Allele-Specific Anti-HLA Antibodies after the Transplantation of a Fully-Matched Kidney Allograft and the Diagnostic Approaches Required for Excluding Harmful Effects after Subsequent Renal Regrafts
}

\author{
Gerald Schlaf*, Jakob Kehlen, Anja Wahle, Diana Mauer, Wolfgang Altermann
}

Tissue Typing Laboratory (GHATT), University Hospital Halle/Saale, Ernst-Grube-Straße 20, 06120 Halle/Saale, Germany; E-Mails: gerald.schlaf@uk-halle.de; kehlenjakob@googlemail.com; anja.wahle@uk-halle.de; diana.mauer@uk-halle.de; hla-labor@uk-halle.de

* Correspondence: Gerald Schlaf; E-Mail: gerald.schlaf@uk-halle.de

Academic Editor: Maurizio Salvadori

Special Issue: Donor Specific Antibody

OBM Transplantation

2021, volume 5 , issue 2

doi:10.21926/obm.transplant.2102142
Received: January 26, 2021

Accepted: May 05, 2021

Published: June 02, 2021

\begin{abstract}
The specification of anti-human leukocyte antigen (HLA) antibodies is an important task for patients awaiting kidney allografts. Especially the patients immunized in previous transplantations, transfusions, or pregnancies must be carefully observed, since grafting patients with HLA antigens/phenotypes recognized by their pre-formed antibodies are the main cause of harmful hyperacute and acute rejection. The complement-dependent lymphocytotoxicity-based de facto (physical) crossmatching (CDC-CM) has thus been implemented as the last diagnostic obstacle before kidney allografting. Here, an assay is performed by incubating the donors' lymphocytes with the sera of the prospective recipients, and a negative outcome was desired for eligibility of the underlying organ allocation. Furthermore, valid antibody specification has to be performed at least quarterly for each patient on the kidney waiting list, as defined by certain guidelines, for example, the Eurotransplant guidelines. Based on the exclusion of these specificities, also referred to as
\end{abstract}

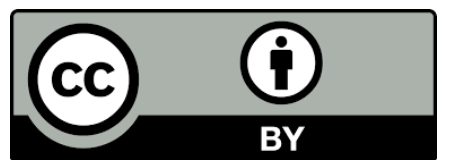

(C) 2021 by the author. This is an open access article distributed under the conditions of the Creative Commons by Attribution License, which permits unrestricted use, distribution, and reproduction in any medium or format, provided the original work is correctly cited. 
virtual crossmatching, certain donors are a priori listed as unacceptable for these recipients. In this case report, we showed that defining unacceptable antigens may be difficult if the recipients' antibodies are allele-specific after being generated in the patient who is expressing the HLA-class II antigen DQ6 and also developing antibodies against this antigen. Low resolution (two-digit) typing is used before kidney allografting. Thus, these antibodies are generally not definable, as donors and recipients share the same antigen (allelic group). Here, we demonstrate the diagnostic approaches required to exclude inadequate kidney donors for a patient exhibiting antibodies only against the HLA-DQB1*06:04 allelic variant and not against the common phenotype HLA-DQ6. In practice, the patient's HLA-class II high resolution (four-digit) typing, as well as his antibody specification at the highest (single antigen) resolution, are included. Furthermore, we critically discuss, according to the Eurotransplant guidelines, the missing possibility to declare own HLA-antigens unacceptable, which may be very helpful for recipients who exhibit allele-specific antibodies.

\section{Keywords}

Allele-specific antibodies; antibody-specification; anti-HLA antibodies; donor-specific antibodies; crossmatching; kidney allografting; Luminex technique; rejection

\section{Introduction}

More than 50 years ago, the correlation between antibodies directed against the antigens of donor tissues and hyperacute rejections of allografts was described for the first time [1]. Later studies provided evidence that these donor-specific antibodies (DSA) are primarily directed against the human major histocompatibility complex (MHC), the human leukocyte (HLA) antigens [2, 3]. In order to prevent kidney recipients from hyperacute and acute rejections, the complementdependent cytotoxicity (CDC) technique was developed 50 years ago and established as a standard technique in the 1970s. In CDC, lymphocytes isolated from a given donor's blood are incubated with the prospective recipient's serum to facilitate a complement-dependent attack after adding complement from rabbits. As the last step before allografting, this assay must have a negative outcome. Else, a DSA-mediated cytotoxic (complement-activating) effect would occur, rendering the donor and recipient incompatible. As donor lymphocytes are directly incubated with the serum of the selected recipients, the test is termed de facto (physical) crossmatching. Crossmatching is performed using CDC-based assays as well as other assays (flow cytometry or solid phase-based) in which whole donor cells or HLA-molecules extracted from the donors are incubated with the recipients' sera to detect a traceable signal or reaction [4-6]. Besides de facto crossmatching, there is a theoretical approach called virtual crossmatching. This approach is generally used before de facto crossmatching right from the time of registration in the kidney waiting list. Virtual crossmatching is performed to predetermine certain HLA-antigens as unacceptable for a given recipient if the person has developed anti-HLA antibodies due to prior transplantations, transfusions, and/or pregnancies. Owing to improvements in antibody assessment through technical advances in the last ten years, virtual crossmatching has gained increasing relevance [7-9]. The first assays performed using this technique consisted of cell trays combining panels of living lymphocytes to 
determine the specificities of merely cytotoxic anti-HLA antibodies. Today, native or recombinant HLA antigens, after their isolation and purification, are adhered to small plastic carrier particles used as solid matrices, and then assessed by the Luminex platform to detect the carrier bead and fluorescence dye-labeled secondary antibodies bound to the primary anti-HLA antibodies [5]. In 1998, it was first suggested to abstain from de facto CDC-based crossmatching for non-immunized patients [10]. Although the idea of implementing virtual crossmatching as a complete replacement for de facto crossmatching had been put forward more than 20 years ago, the wider acceptance of this approach has been accompanied by the progress of Luminex-based techniques, which have increased the resolution up to the level of single-antigen beads (SAB); thus, providing the required number of immobilized single antigen analytes. Although the transplantation programs in some countries use virtual crossmatching as the only evaluation method before allografting for certain groups of recipients, the Eurotransplant community currently performs de facto crossmatching as the last assessment before grafting [11-13]. However, the initial selection of offered kidneys is based on the virtual crossmatching procedure by determining unacceptable antigens to exclude prospective recipients who exhibit the corresponding antibodies. Thus, de facto crossmatching is only performed as a transplant crossmatching by the recipients' transplant centers and not as an allocation crossmatching by the donors' transplant centers. As shown in the case here, and referred to later, the approach of exclusive virtual crossmatching before kidney allografting is still under discussion. We report the case of a patient who received a graft completely matched at the level of low-resolution (two-digit) typing as shown by the HLA A-B-DR-mismatch scheme of $0-0-0$. Despite this favorable match, he exhibited unexpected DSA, resulting in a complete graft loss after nine years. The antibodies were identified as allele-specific after having originated in the patient expressing the same allelic group/phenotype as his donor. Using this example, we show that in certain cases, additional diagnostic approaches are required to correctly select the subsequent regraft. This includes procedures that have originally not been intended for kidney allografting or solid organ transplantations, such as high-resolution (four-digit) typing and antibody specifications at the highest single antigen level.

\section{Case Presentation}

A 23-year-old male patient with end-stage renal insufficiency was HLA-phenotyped and genotyped for HLA-class I and class II antigens (HLA-A3,-; B7,62 (Bw6); Cw3,7; DR13,15; DR51,52; DQ6) when he was registered in the kidney waiting list in February 1999 (Table 1). The quarterly antibody screening runs, which at that time were completely based on CDC-dependent cell typing trays, resulted in panel reactivities (PRA) of $67 \%$ (on average) using in-house CLL (Chronic Lymphatic Leukemia) plates with cells from 30 donors. Using in-house and commercial PBL (Peripheral Blood Lymphocytes) cell trays with cells from 40 to 60 donors, an average of 55\% PRA was recorded. Thus, both types of cell trays showed a significant degree of anti-HLA immunization since registration to the kidney waiting list. This unexpected degree of initial sensitization occurred due to former transfusions received by the patient. Over the 17 quarterly antibody screening runs performed until 2003, when the patient finally received an allograft, the antibody specificities reliably identified using the above-mentioned cell tray analyses were anti-HLA-A2, anti-HLA-A23 (9), anti-HLA-A24 (9), and anti-HLA-B27 (Table 1). Despite using HLA-class II antigen-expressing CLL, it was not possible to reliably identify the corresponding anti-HLA class II specificities. However, it seems highly probable 
that the "hidden" anti-HLA class II specificities were covered by the anti-HLA class I antibodies in the CDC-based analyses conducted in those days. The situation did not change until June 2003, when a deceased donor kidney bearing the HLA-antigens - A3-; B7,62 (Bw6); Cw3,7; DR13,15; DR51,52; and DQ6 - was offered. Importantly, at the level of low-resolution typing, not only the antigens of the HLA-class I gene loci $A$ and $B$, which define the first two positions of the HLA-A-B-DR mismatch scheme, were compatible, but also the $\mathrm{CW}$ antigens showed compatibility (Table 1 ). The HLA-class II DQ antigen DQ6, in addition to the DR-antigens, was also compatible since no second HLA-DQ antigen was presented by the donor to act as an additional transplantation antigen. The situation that was highly favorable for the patient led to the mismatch scheme of HLA A-B-CW-DR-DQ 0-00-0-0 comprising five gene loci HLA A-B-CW-DR-DQ instead of the three loci generally included (HLAA-B-DR). The full HLA compatibility at the low-resolution typing level is provided in Table 1. Neither were any clinical rejection data of the patient sent to our laboratory, nor was there any examination of the patient's antibody status requested by the transplant center or any physician supervising the transplantation course after the transplantation between June 2003 and September 2013.

Table 1 HLA low-resolution typing of the patient and his (deceased) donor from 2003, and the primarily identifiable anti-HLA antibody specificities of the patient's serum.

\begin{tabular}{lll}
\hline Patient's HLA- & Donor's HLA- & anti-HLA Ab specificities \\
Low Res. Typing: & Low Res. Typing: & already known in 2003*: \\
A3,- & A3,- & anti-A2,-A23(9),-A24(9), \\
B7, 62(15) [Bw6] & B7,62(15) [Bw6] & anti-B27 \\
Cw3,7 & Cw3,7 & \\
DR13(6),15(2) & DR13(6),15(2) \\
DR51,52 & DR51,52 & \\
DQ6(1) & DQ6(1) & \\
HLA-class II High Res. & & \\
Typing & & \\
DRB1*13:01,*15:01 (2014 and 2019) & \\
DQB1*06:02, *06:03 (2019) & & \\
\hline
\end{tabular}

Ab.: antibody; res.: resolution; ${ }^{*} \mathrm{CDC} /$ cell tray-based antibody specifications reliably identified between 1999 and 2002; broad antigens are written in parentheses.

Thus, it was a surprise when our laboratory was asked to investigate the re-registered patient on the kidney waiting list in September 2013, after over ten years. Meanwhile, the antibody diagnosis technique had substantially improved by the introduction of several solid-phase-based antibody screening and antibody specification assays $[6,9,10]$. The DynaChip glass microchip technique, introduced in our laboratory in 2008, was the only completely automated antibody specification system and was discontinued by the manufacturer in 2011 for commercial reasons, with the last run on this system conducted in 2012. Hence, afterwards we performed nearly all of the antibody specifications using Luminex single donor (ID) resolution (today distributed by Immucor, Dreieich, Germany) with microbeads on which native HLA class I or class II antigens had been immobilized after being isolated from a single donor. We decided to use this ID system instead of the Single Antigen (SA) system to avoid pseudo-specification of so-called natural antibodies. These antibodies are only directed against cryptic/artificial epitopes of recombinantly generated single HLA-antigens. 
However, they do not bind the corresponding native epitopes of HLA-class I antigens isolated from thrombocytes or the HLA-class II antigens isolated from lymphoma cells [6, 14-17]. Furthermore, the lower single donor (ID) level of resolution, in contrast to the higher resolving single antigen system, provided accurate values of panel reactivity (PRA \%), indicating the degree of immunization and providing the statistical value of the prospective outcome of a recipient's positive crossmatch.

In addition to identifying the anti-HLA-class I antibodies anti-A2, anti-A23(9), anti-A24(9), and anti-B27 (already determined previously by the CDC-based cell tray analyses), it was possible to identify the anti-HLA A68(28) antibodies, which most probably did not represent cytotoxic antibodies (Table 2). Furthermore, several additional antibodies directed against HLA-class II antigens, such as DR7, DR9, DR12(5), DR14(6), and DR18(3), were identified in all subsequent quarterly antibody screening runs between the years 2013 and 2019. The solid phase-based PRAvalues were always around $50 \%$ for both HLA-class I and HLA-class II antigens. Interestingly, clear signals of antibodies directed against the patient's own antigen DR13(6) were observed from the time of re-registration in the waiting list (Table 2). The anti-DR13 values from two independent analyses (third quarterly screening in 2013 and first screening in 2015) indicated that all the beads bearing the allele DRB1*13:03 were strongly positive, whereas all the beads bearing the allele DRB1*13:01 were negative (Table 3). We were aware that the allele-specific antibodies might have been the reason for this, as the patient exhibited antibodies only against the HLA-DRB1*13:03 allelic variant and not against the common phenotype HLA-DR13 (allelic group HLA-DRB1*13). As a plausible control, we performed an HLA-class II DRB1* high resolution (four-digit) typing of the patient using DNA sequencing (Protrans, Ketsch, Germany). The patient's typing results (HLADRB1*13:01, *15:01 and DQB1*06:02,*06:03) showed that his DRB1*13 allelic variant differed from that against which his antibodies had been generated (anti-DRB1*13:03) as hypothesized by us (Table 2 and Table 3).

Table 2 HLA low-resolution re-typing of the patient in 2013 and the HLA-class II highresolution typing results added between 2014 and 2019. Luminex Single Donor/IDdefined antibody specificities are also indicated.

\begin{tabular}{ll}
\hline Patient's HLA Low Res. Typing: & anti-HLA Ab specificities identified \\
& by Luminex Single Donor/ID in 2013: \\
A3,- & anti-A2 [\#],-A23(9) [\#],-A24(9) [\#],-A68(28) \\
B7, 62(15) [Bw6] & anti-B27 [\#] \\
Cw3,7 & \\
DR13(6),15(2) & anti-DR7 [\#],-DR9 [\#],-DR12(5) [\#], \\
DR51,52 & anti-DR13(6),-DR14(6) [\#],-DR18(3) [\#] \\
DQ6(1) & \\
HLA-class II High & \\
Res. Typing: & \\
DRB1*13:01, *15:01 (2014 and 2019) & \\
DQB1*06:02, *06:03 (2019) & \\
\hline
\end{tabular}

Broad antigens are written in parentheses; [\#]: confirmed at the higher single antigen specification level (March 2019 and August 2019) and identified as cytotoxic (August 2019), bold: antigen of the patient and additionally defined as antibody specificity using Luminex single donor assays. 
Table 3 Raw data and the resulting assignments of two quarterly screening runs using Luminex-based HLA class II single donor (single ID) antibody specification kits.

\begin{tabular}{llllll}
\hline Quarter 03/2013 & & & & & \\
\hline Bead ID & Raw Value (MFI) & DR1 & DR2 & DR3 & Assignment \\
129 & $\mathbf{1 5 , 8 3 5}$ & $* 07: 01$ & $* 13: 03$ & $3 * 01: 01$ & positive \\
120 & $\mathbf{1 5 , 3 3 2}$ & $* 11: 02$ & $* 13: 03$ & $3 * 01: 01$ & positive \\
112 & $\mathbf{1 4 , 1 0 4}$ & $* 08: 04$ & $* 13: 03$ & $3 * 01: 01$ & positive \\
$\ldots$ & $\ldots$ & $\ldots$ & $\ldots$ & $\ldots$ & $\ldots$ \\
130 & 00,509 & $* 16: 02$ & $* 13: 01$ & $3 * 02: 02$ & negative \\
115 & 00,459 & $* 01: 01$ & $* 13: 01$ & $3 * 02: 02$ & negative \\
\hline Quarter 01/2015 & & & & & \\
\hline Bead ID & Raw Value (MFI) & DR1 & DR2 & DR3 & Assignment \\
120 & $\mathbf{1 4 , 4 5 0}$ & $* 11: 02$ & $* 13: 03$ & $3 * 01: 01$ & positive \\
112 & $\mathbf{1 3 , 6 6 8}$ & $* 08: 04$ & $* 13: 03$ & $3 * 01: 01$ & positive \\
129 & $\mathbf{8 , 7 6 3}$ & $* 07: 01$ & $* 13: 03$ & $3 * 01: 01$ & positive \\
$\ldots$ & $\ldots$ & $\ldots$ & $\ldots$ & $\ldots$ & $\ldots$ \\
130 & 00,411 & $* 16: 02$ & $* 13: 01$ & $3 * 02: 02$ & negative \\
115 & 00,361 & $* 01: 01$ & $* 13: 01$ & $3 * 02: 02$ & negative \\
\hline
\end{tabular}

MFI: mean fluorescence intensity (raw values); bold: apparent specificity, which was not verifiable using specification at the single antigen level

In kidney allografting, virtual exclusions from allocations are based only on low-resolution typing. Due to the guidelines of Eurotransplant, it was (and still is) impossible to declare own antigens/allelic groups as unacceptable. Thus, an alternative was required to prevent the patient from being offered an organ with the DRB1*13:03 allele. We agreed with the transplant center to perform a high resolution (four-digit) typing of all the offered organs with the DR13 antigen/DRB1*13 allelic groups. We used a sequence-specific primer (SSP) PCR-based typing kit (Olerup, Austria, Germany) between the years 2014 and 2019 to work efficiently during emergency duties. During this period, the patient was listed five times on the allocation table comprising the first ten computer algorithm-selected potential recipients. Until 2019, the patient was not selected to be allografted due to lower positions in the allocation tables or, in one case, due to the withdrawal of the kidney when it turned out to be malignant after its explantation from the donor. Here, we provide only the data with immunological relevance in the current context, and therefore, present the data of a crossmatch outcome that completely changed our strategy to refuse all DRB1*13:03 allele-positive organ offers. None of the five offers expressed any of the unacceptable antigens listed as antibody specificities in Table 2, apart from the DR13 antigen, which could not be blocked. In March 2019, a positive CDCbased crossmatch outcome against an offered kidney was obtained. The donation was PCR-typed DRB1*13:01; thus, it matched the patient's DRB1*13:01 allele and had no additional antigens previously considered unacceptable (listed in Table 2 and Table 3). The CDC-based crossmatching exhibited strong signals directed against HLA-class II antigens, as all sera, including the current one and the six previously collected ones, showed B-cell scores ranging between 6 and 8 (about $80 \%$ of the cells were positive) and PBL scores ranging between 2 and 4 (20\%-30\% of the cells were positive). Isolated T-cells, however, did not show any valid CDC-based crossmatch reaction. The complete HLA-typing results of the donation in March 2019 were: HLA-A1,3; B7,35 (Bw4); Cw4,7; 
DR13(DRB1*13:01),15(DRB1*15:01); DR51, DR52; and DQ6. The HLA-class II high-resolution typing performed later using DNA-sequencing (Protrans, Ketsch, Germany) confirmed the donor's DRB1*13:01 allele. Additionally, the DRB1*15:01 allele was shared by the patient and the donor. The HLA-DQB1* high-resolution typing revealed the alleles DQB1*06:01 and DQB1*06:04. These results provided evidence that the strategy of accepting donation offers of kidneys with the DRB1*13:01 allele and refusing the offers of kidneys with the DRB1*13:03 allele was no longer productive for receiving an adequate kidney allograft. None of the DRB1* allelic variants of the recent donor from March 2019 were the immune targets of the anti-HLA class II antigen-directed CDC-based crossmatch results.

We repeated the patient's antibody specification for the first time using a higher level of singleantigen instead of the single donor resolution (Immucor, Dreieich, Germany). As shown in Table 4, the results were unexpected. None of the DRB1*13 alleles (DRB1*13:01, *13:03, and *13:05) provided any immune target for the patient, which was true for the serum sample taken in March 2019 and the other one taken in August 2019. Thus, the allele-specific antibody was, as erroneously suggested by the single donor (ID) specification kit, not directed against the DRB1*13:03 allele (Table 4). Although, the antibody was directed against a DQB1*06 allele regularly associated with the DRB1*13 allelic group due to the tight linkage disequilibrium between the gene loci DRB1* and DQB1*. In our case, it was shown to be only the DQB1*06:04 allele, as the other DQB1* alleles DQB1*06:01, DQB1*06:02, and DQB1*06:03 did not show any signal (Table 4). It is noteworthy that the alpha-chain of HLA-DQ antigen could be excluded as an immune target, as not only the allele DQA1*01:02 composing the HLA class II complex with DQB1*06:04, but all DQA1* allelic groups investigated in this assay, were in general not involved in any immune response (data not shown). Furthermore, anti-HLA antibodies directed against the antigens of HLA-class II locus DP were absent (data not shown). Thus, the single antigen specification of anti-HLA antibodies, providing the higher level of resolution, allowed to determine the allele-specific anti-DQB1*06:04 antibodies, which were also complement-activating (cytotoxic) as first indicated by the HLA-class II-specific crossmatch outcome in March 2019. Additionally, to confirm the complement-activating capacity of the anti-DBQ1*06:04 antibodies, we combined the single antigen specifications of the two sera (from March 2019 and August 2019) with a supplementary kit detecting the complement cleavage product C3d (Immucor, Dreieich, Germany), to determine the complement-activating capacity of the antibodies. Interestingly, only one of the anti-HLA class I antigen-binding antibodies (Table 2) confirmed at the higher single antigen level was characterized as non-cytotoxic (anti-A68), and thus, was not detected in the former cell tray analysis. Additionally, all the antibodies that bound to the antigens of HLA-class II allelic groups shown in Table 2 (anti-DR7, anti-DR9, anti-DR12, anti-DR14, and anti-DR18) were cytotoxic. Thus, instead of anti-DR13 antibodies (incorrectly showing high specificity) (Table 2 and 3, in bold), antibodies directed against the DQB1*06:04 allele were actually cytotoxic (Table 4, in bold). Due to the complement-binding characteristics of anti-HLA DQB1*06:04 antibodies, we decided not to offer an SSP-PCR-based high-resolution typing for any donor exhibiting the HLA-DQ6 antigen/allelic group to the transplant center, as was previously done for DR13, which had incorrectly been defined as immune target. This decision was taken because i) it was laborious to perform the procedure, in addition to the various applications already required during emergency duties, and ii) anti-DQB1*06:04 antibodies were identifiable using CDC-based crossmatching with strict observations of HLA-class II-specific reactions, as shown by the crossmatch results from March 2019. 
Table 4 Adjusted MFI-data and the resulting assignments of two antibody specification runs using Luminex-based HLA class II single antigen (LSA) specification kits.

\begin{tabular}{lllll}
\hline March 19 & & & & \\
\hline Bead ID & Adj. Value (MFI) & Allele & Antigen & Assignment \\
225 & 00,124 & DRB1*13:01 & DR13(6) & negative \\
226 & 00,193 & DRB1*13:03 & DR13(6) & negative \\
227 & 00,270 & DRB1*13:05 & DR13(6) & negative \\
272 & $\mathbf{8 , 6 0 4}$ & DQB1*06:04 [\#] & DQ6(1) & positive \\
270 & 00,169 & DQB1*06:02 & DQ6(1) & negative \\
271 & 00,243 & DQB1*06:03 & DQ6(1) & negative \\
267 & 00,133 & DQB1*06:01 & DQ6(1) & negative \\
\hline August 19 & & & & \\
\hline Bead ID & Adj. Value (MFI) & Allele & Antigen & Assignment \\
225 & 00,190 & DRB1*13:01 & DR13(6) & negative \\
226 & 00,328 & DRB1*13:03 & DR13(6) & negative \\
227 & 00,360 & DRB1*13:05 & DR13(6) & negative \\
272 & 6,296 & DQB1*06:04 [\#] & DQ6(1) & positive \\
270 & 00,233 & DQB1*06:02 & DQ6(1) & negative \\
271 & 00,344 & DQB1*06:03 & DQ6(1) & negative \\
267 & 00,187 & DQB1*06:01 & DQ6(1) & negative \\
\hline
\end{tabular}

MFI: mean fluorescence intensity (adjusted values); bold: positive allelic antibody specificity; [\#]: identified as cytotoxic (August 2019).

Fortunately, the patient was offered a kidney typed HLA-A26; B8,35 (Bw6); Cw4,7; DR11,15; DR51, DR52; and DQ6,7 in June 2020; thus, ending the process. The current serum sample (April 2020), along with eight previous sera, taken between October 2015 and January 2020, were used, as they had the highest Luminex-Single Donor determined PRA values (between $55 \%$ and $60 \%$ ). All outcomes of the CDC-based crossmatching, including the HLA-class II-indicating B-cell crossmatching, were negative. The antigen DQ6 (allelic group DQB1*06), possibly comprising the problematic allele DQB1*06:04, was again involved due to its tight linkage disequilibrium with the HLA-DR15 antigen (DRB1*15 allelic group). Other specificities (Table 2) of immunological relevance had already been determined as unacceptable antigens. However, since we relied on the CDC-based identification of allele-specific anti-DQB1*06:04 antibodies, the negative CDC-crossmatch result was accepted, and the kidney was allografted. Supplementarily specifying only the relevant alleles, high-resolution typing of the donor's DNA as plausibility control revealed HLA-DRB1*15:01 and DQB1*06:02, thus indicating that no hazardous DQB1*06:04 allele, but one of the patient's DQB1* alleles, had been allografted (Table 2). Since June 2020, for the first eight months, the patient's graft was characterized with stable functions, with no visible signs of rejection. It could be concluded that high-resolution typing results for the relevant antigens of the patient and the donor, in combination with higher level (single antigen) antibody specification, prevented the patient from receiving a hazardous allelic variant of an HLA-antigen/allelic group, which appears to be an auto-antigen that could not have been excluded using low-resolution typing alone. 


\section{Discussion}

It is well known that the compatibility of HLA antigens between a donor and the recipient has an important effect on the short-term and long-term survival of allografted kidneys and other organs $[18,19]$. More than twenty years ago, Opelz and co-workers reported that the ten-year graft survival rate of primary post mortem kidney allografts was about $20 \%$ lower for the allografts characterized by six mismatches (MM A-B-DR $=2-2-2$ ) compared to those characterized by no mismatch (MM A$B-D R=0-0-0)$. In previous studies, comprising more than 150,000 recipients, conducted between the years 1987 and 1997, the impact of HLA-class I gene loci A and B was shown to be equivalent and additive to and that of the class II locus DR [18]. Although graft survival has improved over time due to the increased pharmacological efficiency of rejection treatment, the relative impact of HLAmatching on graft survival was observed to be similar in a five-year analysis between 2000 and 2004 [19]. Interestingly, more accurate DNA typing results, developed in the 1990s, have significantly improved the reliability of HLA-matching and allograft survival [18]. This effect is explained by the higher accuracy of genotyping, which corrected the previous serological errors. Although the three mismatch-defining HLA gene loci $A, B$, and DR are regarded as the most important criteria, an increasing number of studies have discovered the adverse effects of graft survival in pre-sensitized recipients due to other mismatched HLA-loci [20-24]. Research provides evidence that a high degree of compatibility, including all polymorphous HLA-gene loci, is an important aspect for medium-term (one up to five years) and long-term (more than five years) graft survival and exceedingly for successful regrafting.

The first kidney donation for the presented case in 2003 was optimal, as not only were the threemismatch scheme-defining antigens HLA-A, B, and DR (MM =0-0-0) compatible, but those for CW and $D Q$ also showed compatibility. This led to the highest possible level of HLA-compatibility based on low-resolution/two-digit typing (MM A-B-C-DR-DQ =0-0-0-0-0). It is noteworthy that the linkage disequilibrium between the HLA-class I gene loci $B$ and $C$, and between the HLA-class II gene loci $D R$ and $D Q$, does not correctly indicate an association of $C$ and $D Q$ antigens, respectively, in all cases. The linkage disequilibrium between $B$ and $C$ is not very tight, and some DR-antigens (HLADR4, DR7, DR8, and DR13) may be associated with one or the other of two different HLA-DQ antigens, which is not reflected by a mismatch scheme comprising only the three gene loci HLA-A, $B$, and DR. However, despite the highest possible degree of two-digit HLA-matching, the recipient developed antibodies, eventually causing him to lose the allograft and re-enter the kidney waiting list after ten years.

As shown here, the situation was complicated by former blood transfusions. In addition to some anti-HLA class I antibodies known previously (Table 1), the improved solid-phase/Luminex-based antibody specifications led to a reliable identification of an additional HLA-class I specificity and of several anti-HLA class II specificities (Table 2), compared to the old and inefficient cell tray analysis (Table 1). Unexpectedly, one specificity (DR13) was also expressed by the recipient and thus indicated allele-specific antibodies. However, the Luminex-based specification assays at the single donor (ID) level had initially failed to identify the correct specificity. Thus, initially, the allele DRB1*13:03 was falsely identified as an immune target and excluded by additional PCR-based highresolution assays. However, through the unexpected and accidental positive B-cell crossmatch result against a donor exhibiting DRB1*13:01, it became evident that none of the allelic variants of DR13/DRB1*13 were immune targets; instead, the immune target was an associated allelic variant 
of DQ6/DQB1*06 called DQB1*06:04. This specificity, however, could only be identified using the higher level of Luminex-based single antigen specification.

This case provides evidence that when allele-specific antibodies are involved, antibody specification assays providing a higher level of resolution are required, although their antigens may be characterized by cryptic epitopes of some recombinantly generated single antigens, and thus, show false (so-called natural) antibodies. It has been known for many years that these antibodies are not directed against the corresponding native antigens. Furthermore, weak DSA, indicated by virtual Luminex-based single antigen analysis, have repeatedly been shown to be irrelevant for graft survival [6, 14-17, 25-29]. These important aspects have been the focus of the Luminex-based single antigen specifications in the last decade and have limited the clinical value of exclusive virtual crossmatching. Prospective recipients may be excluded from too many offers of organs without real DSA-based contraindications. Considering these aspects, we decided to continue our antibody specifications by initially using the native antigen-based single donor (ID) specification assays. In cases with higher degrees of immunization that caused overlapping and unidentifiable single antibody specificities, we generally use single antigen-based analysis as the second step. However, we learned from the present case to strictly perform single antigen specification whenever allelespecific antibodies are suspected, as the identification of these antibodies may be beyond the limits of the single donor (ID) assay.

Our data is supported by a recent publication showing certain interlocus eplets as immunodominant epitopes, which are defined by distinct polymorphous amino acid residues [30]. According to Duquesnoy and Marrari (2020), the difference between HLA-DQB1*06:01/02/03 (amino acid residue 57D/eplet rqp57D) and HLA-DQB1*06:04 (amino acid residue 57V/eplet rq57V) was predicted to lead to different epitopes, which strongly supports our finding of an allele-specific anti-DQB1*06:04 antibody by a recipient expressing the alleles DQB1*06:02 and DQB1*06:03 [30]. Thus, our data support the "HLA-matchmaker" molecular modeling concept, proposed in 2007, by defining the eplets, along with their surrounding region within $3.5 \dot{A}$, as products of polymorphous residues [31]. Our data practically verified the theoretical DQB1*06:04-specific epitope difference in the HLA-DQB1*06 allelic group [30].

One important aspect should be discussed, which arises from the fact that allele-specific antibodies are directed against a variant of the allelic group expressed by the recipient. Thus, based on virtual crossmatching after an exclusive low-resolution typing of a given recipient, these antibodies appear to be autoreactive. Consequently, as per the guidelines of Eurotransplant (ET), it is impossible to state that the "own" respective antigens/allelic groups (DQ6) are unacceptable. Similar cases have been described in other studies, where the recipient developed antibodies against HLA-DRB3*02:02 and DQB1*06:03, although the graft, which had been the recipient's first, was identical for HLA-class II loci DR and DQ at the level of low-resolution typing. High-resolution typing, however, revealed five allelic incompatibilities between the donor and the recipient [32].

Another case from our laboratory highlights the benefit of blocking own antigens/allelic groups [33]. A recipient expressing the rare HLA-class I $A * 25: 14$ allele developed antibodies not only against the phenotype HLA-A25/allelic group HLA-A*25 but also against the complete underlying broad antigen HLA-A10. All the split antigens HLA-A25, A26, A34, and A66 share the common (broad) HLAA10 epitope, which is found frequently among donors. Despite this detrimental situation, it was not possible to block the broad HLA-A10 antigen as it was irreversibly defined as an autoantigen in the allocation guidelines of Eurotransplant. Nearly all of the offered organs expressed at least one of 
the HLA-A10 broad antigens, as these antigens define the level of HLA-class I matching. In order to a priori prevent the patient from most of those harmful antigens by blocking all the HLA-A10 split antigens, we removed the HLA-A25 antigen from the recipient's typing results. Fortunately, as in the case presented here, the anti-HLA A10 antibodies were cytotoxic, i.e., they were also detectable using CDC-based crossmatching. Also in that historical situation, the de facto crossmatching prevented the recipient from receiving harmful antigens.

As discussed by Morris and co-workers two years ago, exclusive virtual crossmatching may be advantageous, especially for immunologically inconspicuous recipients, for reducing harmful ischemia during allografting [9]. This approach, however, is currently not feasible for laboratories under the supervision of Eurotransplant, where HLA-class I matching is performed based on broad antigens, i.e., at the lowest possible level of HLA-histocompatibility. At least HLA-class II matching is, in contrast, based on the level of split antigens. Both levels, however, may be insufficient, as shown by current and previous cases.

\section{Conclusion}

As previously highlighted by Proust and co-workers [32], we argue that the definition of antibody epitopes, according to the eplet (HLA-matchmaker) concept, should be an essential requirement and the basis for a reliable virtual crossmatching. The approach of exclusive virtual crossmatching needs to be critically analyzed for the recipients' safety if low-resolution (two-digit) typing and broad antigen matching are used for determining only the lowest level of HLA compatibility [34]. Especially for patients who wait for regrafting, this approach seems irrational, as evidenced by an increasing number of individual cases.

\section{Author Contributions}

AW, DM and JK collected the data. GS, JK and WA analyzed the data. GS prepared the manuscript.

\section{Competing Interests}

The authors have declared that no competing interests exist.

\section{References}

1. Patel R, Terasaki PI. Significance of a positive crossmatch test in kidney transplantation. $\mathrm{N}$ Engl J Med. 1969; 280: 735-739.

2. Ahern AT, Artruc SB, Della Pelle P, Cosimi AB, Russel PS, Colvin RB, et al. Hyperacute rejection of HLA-AB-identical renal allografts associated with $B$ lymphocyte and endothelial reactive antibodies. Transplantation. 1982; 33: 103-106.

3. Chapman JR, Taylor C, Ting A, Morris PJ. Hyperacute rejection of a renal allograft in the presence of anti-HLA Cw5 antibody. Transplantation. 1986; 42: 91-93.

4. Lobo PI, Spencer C, Gorman J, Pirsch G. Critical appraisal of complement dependent microlymphocytotoxicity assay for detecting donor-specific alloantibody pretransplantimportance of indirect immunofluorescence as a superior alternative. Hum Immunol. 1981; 2: 55-64. 
5. Altermann W, Seliger B, Sel S, Wendt D, Schlaf G. Comparison of the established standard complement-dependent cytotoxicity and flow cytometric crossmatch assays with a novel ELISAbased HLA crossmatch procedure. Histol Histopathol. 2006; 21: 1115-1124.

6. Schlaf G, Pollok-Kopp B, Altermann W. Sensitive solid-phase detection of donor-specific antibodies as an aid highly relevant to improving allograft outcomes. Mol Diagn Ther. 2014; 18 : 185-201.

7. Amico P, Hönger G, Steiger J, Schaub S. Utility of the virtual crossmatch in solid organ transplantation. Curr Opin Organ Transplant. 2009; 14: 656-661.

8. Jackson AM. The virtual crossmatch: An essential tool for transplanting sensitized patients. Clin Transpl. 2014; 16: 131-136.

9. Morris AB, Sullivan HC, Krummey SM, Gebel HM, Bray RA. Out with the old, in with the new: Virtual versus physical crossmatching in the modern era. HLA. 2019; 94: 471-481.

10. Kerman RH, Susskind B, Ruth J, Katz S, Van Buren CT, Kahan BD. Can an immunologically, nonreactive potential allograft recipient undergo transplantation without a donor-specific crossmatch? Transplantation. 1998; 66: 1833-1834.

11. Taylor CJ, Kosmoliaptsis V, Sharples LD, Prezzi D, Morgan CH, Key T, et al. Ten-year experience of selective omission of the pre-transplant crossmatch test in deceased donor kidney transplantation. Transplantation. 2010; 89: 185-193.

12. Böhmig GA, Fidler S, Christiansen FT, Fischer G, Ferrari P. Transnational validation of the Australian algorithm for virtual crossmatch allocation in kidney paired donation. Hum Immunol. 2013; 74: 500-505.

13. Turner D, Battle R, Akbarzad-Yousefi A, Little AM. The omission of the "wet" pre-transplant crossmatch in renal transplant centres in Scotland. HLA. 2019; 94: 3-10.

14. Morales-Buenrosto LE, Terasaki PI, Marino-Vazques A, Lee JH, El Awar N, Alberu J. "Natural" human leukocyte antigen antibodies found in nonalloimmunized healthy males. Transplantation. 2008; 86, 1111-1115.

15. Pereira S, Perkins S, Lee JH, Shumway W, LeFor W, Lopez-Cepero $M$, et al. Donor-specific antibody against denatured HLA-A1: Clinically nonsignificant? Hum Immunol. 2011; 72: 492498.

16. Poli F, Benazzi E, Innocente A, Nocco A, Cagni N, Gianatti A, et al. Heart transplantation with donor-specific antibodies directed toward denatured HLA*02:01: A case report. Hum Immunol. 2011; 72: 1045-1048.

17. Otten HG, Verhaar MC, Borst HP, van Eck M, van Ginkel WG, Hene RJ, et al. The significance of pretransplant donor-specific antibodies reactive with intact or denatured HLA in kidney transplantation. Clin Exp Immunol. 2013; 173: 536-543.

18. Opelz G, Wujciak T, Döhler, B, Scherer S, Mytilineos J. HLA compatibility and organ transplant survival. Collaborative Transplant Study. Rev Immunogenet. 1999; 1: 334-342.

19. Opelz G, Döhler B. Effect of human leukocyte antigen compatibility on kidney graft survival: comparative analysis of two decades. Transplantation. 2007; 84: 137-143.

20. Frohn C, Fricke L, Puchta JC, Kirchner $\mathrm{H}$. The effect of HLA-C matching on acute renal transplant rejection. Nephrol Dial Transplant. 2001; 16: 355-360.

21. Rees MT, Darke C. HLA-A, B, C, DRB1, DQB1 matching heterogeneity in 'favourable matched' kidney recipients. Transpl Immunol. 2003; 12: 73-78. 
22. Sasaki N, Idica A. The HLA-matching effect in different cohorts of kidney transplant recipients: 10 years later. Clin Transplant. 2010; 25: 261-281.

23. Tran HT, Döhler B, Heinold A, Scherer S, Ruhenstroh A, Opelz G. Deleterious impact of mismatching for human leukocyte antigen $C$ in presensitized recipients of kidney transplants. Transplantation. 2011; 92: 419-425.

24. Ntokou IS, Iniotaki AG, Kontou EN, Darema MN, Apostolakis MD, Kostakis AG, et al. Long-term follow up for anti-HLA donor-specific antibodies postrenal transplantation: High immunogenicity of HLA class II graft molecules. Transpl Int. 2011; 24: 1084-1093.

25. Grenzi PC, de Marco R, Silva RZ, Campos EF, Gerbase-DeLima M. Antibodies against denatured HLA class II molecules detected in Luminex-single antigen assay. Hum Immunol. 2013; 74: 13001303.

26. Visentin J, Guidicelli G, Moreau JF, Lee JH, Taupin JL. Deciphering allogeneic antibody response against native and denatured HLA epitopes in organ transplan-tation. Eur J Immunol. 2015; 45: 2111-2121.

27. Adebiyi OO, Gralla J, Klem P, Freed B, Davis S, Wiseman AC, et al. Clinical significance of pretransplant donor-specific antibodies in the setting of negative cell-based flow cytometry crossmatch in kidney transplant recipients. Am J Transplant. 2016; 16: 3458-3467.

28. Kwon H, Kim YH, Choi JY, Shin S, Jung JH, Park SU, et al. Impact of pretransplant donor-specific antibodies on kidney allograft recipients with negative flow cytometry crossmatches. Clin Transplant. 2018; 32: e13266.

29. Buttigieg J, Ali H, Sharma A, Halawa A. Positive luminex and negative flow cytometry in kidney transplantation: A systematic review and meta-analysis. Nephrol Dial Transplant. 2019; 34: 1950-1960.

30. Duquesnoy RJ, Marrari M. Eplet-based HLA class II matching for transplantation: Design of a repertoire of interlocus eplets shared between HLA-DR, -DQ and-DP alleles. OBM Transplant. 2020; 4. DOI: 10.21926/obm.transplant.2001099.

31. Duquesnoy RJ, Askar M. HLA matchmaker: A molecular based algorithm for histocompatibility determination V. Eplet matching for HLA-DR, HLA-DQ and HLA-DP. Hum Immunol. 2007; 68: 1225.

32. Proust B, Kennel A, Ladriere M, Kessler M, Perrier P. Unexpected anti-HLA-DR and -DQ alloantibodies after nephrectomy of an HLA-DR and DQ identical first renal transplant. Transpl Immunol. 2009; 21: 166-168.

33. Schlaf G, Radam C, Wahle A, Altermann WW. Generation of donor-specific anti-human leukocyte antigen antibodies after the transplantation of a fully matched kidney allograft and its impact on the selection of a subsequent renal regraft. Transplant Proc. 2012; 44: 1442-1445.

34. Arnold ML, Geithner M, Herber M, Kloecker S, Lauer B. Defining the mismatch: What is the meaning of a "reduced match" for the definition of donor-specific anti-HLA antibodies? Proceedings of the 18th annual conference of the German Society for Immunogenetics; 2010 August 26-28; Wien, Austria. 


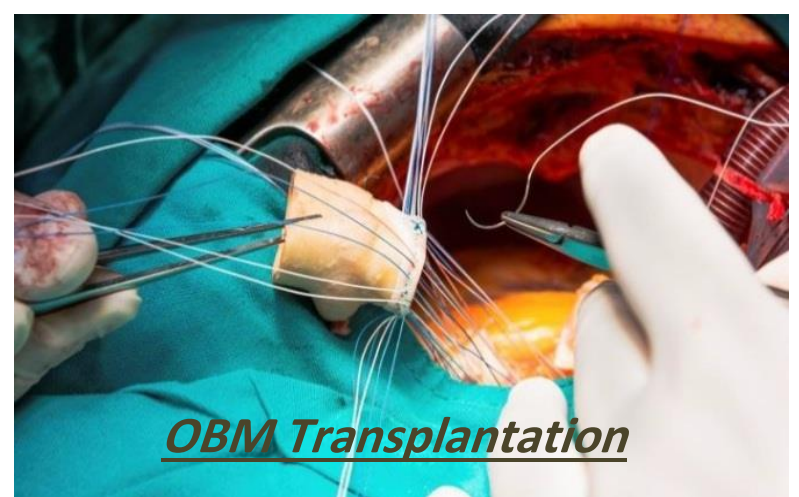

Enjoy OBM Transplantation by:

1. Submitting a manuscript

2. Joining in volunteer reviewer bank

3. Joining Editorial Board

4. Guest editing a special issue

For more details, please visit:

http://www.lidsen.com/journals/transplantation 